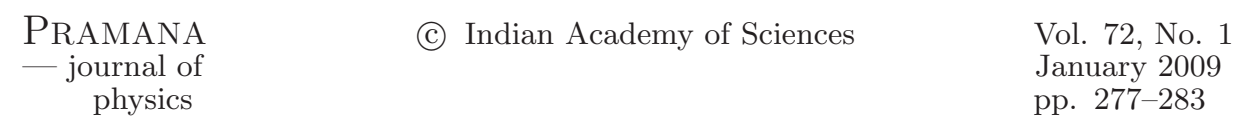

\title{
Working group report: Quantum chromodynamics sub-group
}

Coordinator: ASMITA MUKHERJEE ${ }^{7, *}$

Working group members: R Basu ${ }^{1}$, H Dahiya ${ }^{2}, \mathrm{~L}_{\text {Gamberg }}^{3}, \mathrm{R}_{\text {Godbole }}{ }^{10}$, S Gupta ${ }^{4}$, M C Kumar ${ }^{5}$, L Magnea ${ }^{6}$, P Mathews ${ }^{5}$, N Mathur ${ }^{4}$, A Mukherjee ${ }^{7}$, P J Mulders ${ }^{8}$, V Ravindran ${ }^{9}$ and A Tripathi ${ }^{9}$

${ }^{1}$ The Institute of Mathematical Sciences, Taramani, C.I.T. Campus, Chennai 600113 , India

${ }^{2}$ Department of Physics, Dr. B.R. Ambedkar National Institute of Technology,

Jalandhar 144 001, India

${ }^{3}$ Division of Science, Penn State-Berks Lehigh Valley College, Reading,

Pennsylvania 19610, USA

${ }^{4}$ Tata Institute of Fundamental Research, Homi Bhabha Road, Mumbai 400 005, India

${ }^{5}$ Theory Division, Saha Institute of Nuclear Physics, Sector 1, Block AF, Bidhan Nagar, Kolkata 700 064, India

${ }^{6}$ Dipartimento di Fisica Teorica, Universita di Torino and INFN, Sezione di Torino,

Via P. Giuria, I-10125 Torino, Italy

${ }^{7}$ Physics Department, Indian Institute of Technology Bombay, Powai, Mumbai 400076 , India

${ }^{8}$ Department of Physics and Astronomy, VU University, NL-1081 HV Amsterdam,

The Netherlands

${ }^{9}$ Harish-chandra Research Institute, Chhatnag Road, Jhunsi, Allahabad 211 019, India

${ }^{10}$ Centre for High Energy Physics, Indian Institute of Science, Bangalore 560 012, India

${ }^{*}$ Corresponding author. E-mail: asmita@phy.iitb.ac.in

Abstract. This is the report of the QCD working sub-group at the Tenth Workshop on High Energy Physics Phenomenology (WHEPP-X).

Keywords. Single spin asymmetries; intrinsic transverse momentum; resummation.

PACS Nos 13.88.+e; 12.38.-t; 25.40.Ep

\section{Introduction}

The participants of the QCD working sub-group were N Agarwal, S Arunagiri, R Basu, H Dahiya, L Gamberg, R Godbole, S Gupta, M C Kumar, L Magnea, H S Mani, P Mathews, N Mathur, A Mukherjee, P Mulders, V Ravindran and A Tripathi and the coordinator was A Mukherjee. 
The main topics discussed in the working group (WG) were: (1) resummation: this is an essential tool to extend the predictive power of QCD by doing an all-order summation of large logarithmic contributions. In the WG, there were a series of introductory lectures on resummation by L Magnea. The lecture notes together with some exercises can be found in [1].

Another main topic discussed was (2) single spin asymmetries, in particular the role of intrinsic transverse momentum $k_{\mathrm{T}}$-dependent distribution and fragmentation functions. Interesting issues regarding the time reversal properties, universality and factorization were discussed in this workshop. An introduction to these topics can be found in [2].

There were two plenary talks: (1) Non-collinearity in high energy scattering process by $\mathrm{P}$ Mulders. (2) All order results in $Q C D$ by $\mathrm{L}$ Magnea. Besides, there was one non-overlapping WG talk: Generalized transversity: Transverse momentum and spin correlations in $Q C D$ by L Gamberg.

These talks are published separately in these proceedings. There were also working group talks by H Dahiya, M C Kumar, A Tripathi and N Mathur. Below are the abstracts of these talks.

\title{
2. Working group talks
}

\subsection{Surprises in charmonia}

\author{
Nilmani Mathur \\ (joint session with collider WG)
}

Recently there has been tremendous activities in charmonium $(c \bar{c})$ physics. With an extensive large data obtained from $B$-factories (at SLAC, Cornell, KEK and BES) various states with $c \bar{c}$ flavours have been reported to be discovered [3]. Among these, most interesting are the following three states: $X(3872), Y(4260)$ and $Z^{+}(4430)$ (many other states also do not have clear understanding). $X(3872)$ was discovered by Belle (2003) in the reaction $B \rightarrow K X \rightarrow K \pi \pi J / \Psi$ and was subsequently verified by Babar, CDF and D0 experiments. Due to its given mass, width and $J^{\mathrm{PC}}$, it is impossible to associate this state with a $c \bar{c}$ state and naturally theorists speculated it as a more exotic state. However, some of its properties exclude it as a hybrid state and more convincing explanation comes by speculating it as a $D^{0}-\bar{D}^{* 0}$ molecular state or a $(\bar{c} \bar{q})_{3}(q c)_{\overline{3}} /(\bar{c} \bar{c})_{8}(\bar{q} q)_{8}$ tetraquark state or even possibly as a threshold cusp. The state $Y(4260)$ was discovered by Babar (2005) in radiation returned $s$-channel annihilation: $e^{+} e^{-} \rightarrow \gamma_{\mathrm{ISR}} J / \Psi \pi \pi$, with a mass $4259(8)(5)$ and width $88(23)(5) \mathrm{MeV}$ and possible $J^{\mathrm{PC}}=1^{--}$. Similar to $X(3872)$, it is also impossible to identify this state as a simple $c \bar{c}$ state due to the unavailability of a missing $c \bar{c}$ state with that quantum number at that mass region. Due to its large width, small leptonic coupling, small $B$-decay branching fraction and non-observation of identical meson decay modes, and above all, lattice QCD results on that mass region helps to identify it as a $\bar{c} g c$ hybrid state. However, this identification is not conclusive yet and one needs more experimental data. Theoretically one of the major activities should come from lattice QCD. However, studying these states on lattice is quite non-trivial as one needs to separate out threshold decay states which may lie very close to the resonance state. Using an extended set of lattice operators 
one should study the rich spectrum in this area, and such preliminary study has recently been reported [4].

\title{
2.2 Diphoton production at LHC in the unparticle scenario
}

\author{
A Tripathi and M C Kumar \\ (joint session with BSM WG)
}

We studied diphoton production at LHC in unparticle scenario, with scalar and tensor unparticles in the intermediate propagators. The study was done at leading order (LO) in strong coupling and various differential cross-sections were obtained. We also studied the effects of coupling variations. Diphoton production at LHC in unparticle scenario was investigated with next to leading order (NLO) QCD corrections using phase space slicing method. It is found that the $K$-factor (ratio of NLO and LO cross-sections) is large in addition to being dependent on experimental cuts. Stability of the standard model NLO cross-section against the variation of the phase space slicing parameter is presented with the idea of computing the NLO QCD corrections to the diphoton production via spin-2 unparticle at LHC. Spin-2 unparticle contribution at NLO is presented analytically.

This work is done with $\mathrm{V}$ Ravindran and $\mathrm{P}$ Mathews.

\subsection{Chiral constituent quark model and strangeness in the nucleon}

\section{Harleen Dahiya}

Several spin and flavour-dependent parameters characterizing the strangeness content of the nucleon have been calculated in the chiral constituent quark model with configuration mixing $\left(\chi \mathrm{CQM}_{\text {config }}\right)[5,6]$ which is known to provide a satisfactory explanation of the 'proton spin problem' and related issues. It is able to give a quantitative description of the important parameters such as $\Delta s$, the weak axial vector couplings $\Delta_{8}$ and $\Delta_{0}$, strangeness contribution to the magnetic moment $\mu(p)^{s}$, the strange quark flavour fraction $f_{s}$, the strangeness-dependent ratios $2 \bar{s} / u+d$ and $f_{3} / f_{8}$ etc. In the case of $\mu(p)^{s}$, our result is in full agreement with the latest measurement [7] as well as with the lattice QCD calculations [8]. At the leading order constituent quarks and the weakly interacting Goldstone bosons constitute the appropriate degrees of freedom in the non-perturbative regime of QCD and the 'quark sea' generation in the $\chi \mathrm{CQM}_{\text {config }}$ through the chiral fluctuation is the key in understanding the strangeness content of the nucleon.

\section{Discussions and problems studied/proposed}

3.1 Eikonal approximation and power corrections for event shapes

L Magnea, R Basu, H Dahiya, A Tripathi, M C Kumar, L Gamberg, S Arunagiri, $\mathrm{P}$ Mathews and V Ravindran 
Soft gluon effects can be controlled at the perturbative level through resummations, and induce power-suppressed effects which can be studied by using renormalon techniques, or by looking at the ambiguity originated by the Landau pole when the perturbative expansion is summed to all orders. Renormalon and resummation techniques have been unified into a consistent QCD-inspired model of power corrections with the method of dressed gluon exponentiation (DGE) [9]. The basic calculation that has to be performed to study an observable in DGE is the computation of the spectrum of the observable (say, an event shape), at one loop, with the emission of a soft gluon with non-vanishing virtuality. This spectrum can then be used as kernel for exponentiation, resulting in a cross-section which is perturbatively resummed and consistently includes a set of QCD-motivated power corrections.

The calculation of the DGE kernel is often intricate (see for example [10]), while the result for at least the leading set of power corrections is quite simple. One is led to conclude that some further approximation might simplify the calculation, but still get the leading corrections. The proposed approximation is the eikonal approximation: it is known to be exact at leading power for soft gluons, while it is not sufficient for NLL resummation because it misses some important hard collinear effects. These however are expected to contribute only to subleading power corrections.

I propose the following study to test these ideas:

- As a preliminary exercise, (re)compute the thrust distribution at one loop, first for a massless gluon and then for a 'massive' gluon.

- Do the same in the eikonal approximation, verifying that the leading singularities at $T \rightarrow 1$ are reproduced.

- Use the massive eikonal calculation to perform DGE, and compare with the results reported, say, in [10]. Check that leading power corrections are reproduced, and study the discrepancy for subleading ones, with an eye to identify its source.

This study could, with some work, be generalized to other event shapes and more general observables, providing a much simplified method to compute the most phenomenologically relevant power-correction effects.

\subsection{Soft gluon resummation in conformal theories}

\section{Magnea and V Ravindran}

Recent work in the context of $\mathcal{N}=4$ supersymmetric Yang-Mills (SYM) theory and string theory has displayed unexpected properties and applications of the resummation of infra-red and collinear singularities of amplitudes. This resummation, in fact, is not a specific feature of QCD, but it applies to any massless gauge theory. Furthermore, resummation is expressed in terms of exponentiating functions of the running coupling: in a conformal theory, like $\mathcal{N}=4$ SYM, the running coupling is a constant, and integrals over the scale of the coupling become essentially trivial. This 'hidden simplicity' of the perturbative expansion is quite likely tied to the nature of the strong coupling limit of $\mathcal{N}=4 \mathrm{SYM}$, which is expected to be described 
by the AdS/CFT correspondence (for a recent review of this fast-developing field, see $[11])$.

These developments motivate further study of resummations (and in general of the structure of infra-red and collinear singularities) in the conformal limit. A possible avenue of exploration which does not involve extensive calculations or highly innovative techniques might be the following:

- While the AdS/CFT correspondence is particularly simple in the planar $\left(N_{\mathrm{c}} \rightarrow \infty\right)$ limit, results for the infra-red structure of amplitudes are available, for QCD as well as $\mathcal{N}=4 \mathrm{SYM}$, for any $N_{\mathrm{c}}$. Studying the conformal limit of the exponentiation of singularities, in the non-planar case, could uncover some new interesting structures. Such a calculation should begin with, say, the four-point amplitude, for which two-loop resummed results are available [12] in QCD. Non-planar corrections to amplitudes in $\mathcal{N}=4 \mathrm{SYM}$ correspond to string-loop corrections to the AdS/CFT correspondence, and are expected to be very hard to compute in string theory.

\subsection{Soft gluon resummation beyond leading power}

\section{Magnea, R Basu, H Dahiya, A Tripathi, M C Kumar, S Arunagiri and V Ravindran}

There is increasing evidence that it may be possible to extend threshold resummation techniques beyond leading power in the threshold variable (in Mellin space, beyond leading power in $N$ ). For example, a modified DGLAP evolution equation has been proposed in [13], which seems to control soft gluon effects in parton evolution down to $1 / N$ level (leading effects being $\log N$ in this case). The evidence is sparse but nontrivial, the issue has never been systematically tackled: can one go one power beyond the eikonal approximation and still keep some control, some degree of factorization? Note that in QED, Low's theorem does indeed suggest that soft photon radiation is largely determined by the classical brehmsstrahlung spectrum. In this case a relatively simple project would be to study the origin of $1 / N$ terms in a well-understood cross-section at finite orders in perturbation theory, and compare the results with existing factorization theorems and evolution equations, as well as with less established speculations.

- Consider, for example, the Drell-Yan process in the $\overline{\mathrm{MS}}$ factorization scheme. (Re)compute the one-loop cross-section in moment space carefully tracing the origin of soft and collinear gluon effects down to $1 / N$ accuracy. At this order they should all be traceable to subleading terms in the splitting function.

- Repeat the exercise at two loops, considering only the double real gluon emission diagrams. Trace the resulting terms to either subleading collinear effects, or to the phase space effects described in [13]. Examine the possible remainders both in terms of their origin and in terms of their impact on the cross-section.

Note that this kind of exercise also has potential phenomenological application to LHC processes, not only strictly of Drell-Yan type, but also, say, to the case of Higgs boson production via gluon fusion. 
3.4 Model studies of $k_{\mathrm{T}}$-dependent distribution and fragmentation functions: Gluonic pole contribution

P Mulders, A Mukherjee and L Gamberg

The non-vanishing of gluonic pole matrix elements can explain the appearance of single spin asymmetries in high-energy scattering processes. We use a spectator model approach to investigate the spectral properties of quark-quark-gluon correlators and use this to study gluonic pole matrix elements. Such matrix elements appear in principle both for distribution functions (such as Sivers function) and fragmentation functions such as Collins function. We find that for a large class of spectator models, the contribution of gluonic pole matrix element in fragmentation functions vanishes. This outcome is important in the study of universality for fragmentation functions and confirms the findings using a different approach.

This work has been completed and the resulted paper can be found in [14].

3.5 Relations between twist-two and twist-three gauge invariant $k_{\mathrm{T}}$-dependent correlators using equations of motion and Lorentz invariance

P Mulders, A Mukherjee, L Gamberg and H S Mani

Completely unintegrated quark-gluon correlator is gauge invariant if a path ordered exponential (gauge link) is included in its definition. This is parametrized satisfying hermiticity, parity and time reversal. Integrating over components of $k$, one gets the $k_{\mathrm{T}}$-dependent correlator. Constraints from Lorentz invariance and equations of motion give connections between different functions appearing in the parametrization at each step. We propose to investigate these connections including the gauge link, which will connect various $k_{\mathrm{T}}$-dependent distribution and fragmentation functions.

\subsection{Heavy quarkonium production and acollinear physics}

R Godbole, P Mulders, A Mukherjee, L Gamberg and H Dahiya

The formalism for treating non-collinearity of particles as studied for DIS, DY involve an understanding of $k_{\mathrm{T}}$-dependent distribution and fragmentation functions. We propose to study (1) the azimuthal asymmetry of $\gamma$ in $p p^{\uparrow} \rightarrow \gamma+$ jet as a probe of $k_{\mathrm{T}}$-dependent functions; (2) study $p p \rightarrow \Lambda+$ jet; process and polarization of lambda; (3) production of $\mathrm{J} / \psi: p p^{\uparrow} \rightarrow J / \psi+$ jet, and use the azimuthal asymmetries as probe of $k_{\mathrm{T}}$-dependent functions; (4) study $J / \psi$ production at large $p_{\mathrm{T}}$. It is known that in colour octet model, gluon fragmentation contribution is large at large $p_{\mathrm{T}}$, and a probe of $k_{\mathrm{T}}$-dependent fragmentation can be to study polarization of $J / \psi$ in $p p \rightarrow J / \psi+X$. 
Working group report: Quantum chromodynamics sub-group

3.7 Uncertainities in collider predictions

Sourendu Gupta

(Joint session with collider, flavour and BSM WG)

This discussion will be reported in the WG report of the collider WG.

\section{References}

[1] See the website http://www.to.infn.it/ magnea/parlez_en.pdf

[2] See the website http://www.nat.vu.nl/ mulders/

[3] For review, see M. Voloshin, arXiv:0711.4556

E Swanson, Phys. Rep. 429, 243 (2006)

[4] J Dudek, R Edwards, N Mathur and D Richards, Phys. Rev. D77, 034501 (2008)

[5] S Weinberg, Physica A96, 327 (1979)

A Manohar and H Georgi, Nucl. Phys. B234, 189 (1984)

E J Eichten, I Hinchliffe and C Quigg, Phys. Rev. D45, 2269 (1992)

[6] H Dahiya and M Gupta, Phys. Rev. D66, 051501(R) (2002); ibid. 67, 074001 (2003); 67, $114015(2003)$

[7] HAPPEX Collaboration: K A Aniol et al, Phys. Lett. B509, 211 (2001); ibid. Phys. Rev. Lett. 98, 032301 (2007)

[8] D B Leinweber, S Boinepalli, I C Cloet, A W Thomas, A G Williams, R D Young, J

B Zhang, J M Zanotti and J B Zhang, Phys. Rev. Lett. 94, 212001 (2005)

C S An, D O Riska and B S Zou, Phys. Rev. C73, 035207 (2006).

[9] E Gardi, Nucl. Phys. B622, 365 (2002)

[10] E Gardi and L Magnea, J. High Energy Phys. 0308, 030 (2003)

[11] L J Dixon, arXiv:0803.2475 [hep-th]

[12] S Mert Aybat, L J Dixon and G Sterman, Phys. Rev. D74, 074004 (2006)

[13] Yu L Dokshitzer, G Marchesini and G P Salam, Phys. Lett. B634, 504 (2006)

[14] L Gamberg, A Mukherjee and P Mulders, arXiv:0803.2632 [hep-ph] 